\title{
A FIRST STEP TOWARDS A FUZZY FRAMEWORK FOR ANALYZING COLLECTIONS OF JSON DOCUMENTS
}

\author{
Giuseppe Psaila and Stefania Marrara \\ University of Bergamo - DIGIP \\ Viale Marconi 5 - 24044 Dalmine (BG), Italy
}

\begin{abstract}
JSON is a light-weight format for data interchange on the Web, widely adopted by most portals to make their data collections accessible through APIs, each one providing their own data structures. The consequence is that different data sets, even related to the same domain, may have different structures, even though they describe the same information. Thus, analysts must collect and face heterogeneous data structures during the analysis process. Although JSON stores are schema free by definition, their query languages still lack suitable constructs for flexibly querying heterogeneous repositories of JSON documents by expressing linguistic and/or imprecise predicates. In this work, we try to define a first proposal for a flexible query language, based on J-CO-QL, aimed at exploiting the flexibility offered by Fuzzy Set Theory to exploit imprecise (linguistic) concepts or to deal with uncertainty. J-CO-QL is the query language of the J-CO framework, designed to query and transform large and heterogeneous JSON data sets.
\end{abstract}

\section{KEYWORDS}

Collections of JSON Documents, JSON Document Stores, Flexible Query Language, Fuzzy Set Theory

\section{INTRODUCTION}

JSON (JavaScript Object Notation) is a light-weight format for data interchange on the Web. JSON is completely language independent, and it is easy to understand and generate. In the last years, JSON has been preferred to XML (the eXtensible Mark-up Language) for several reasons: JSON does not use tags, is shorter, quicker to read and write, and can use arrays. Furthermore, an XML document is represented in main memory by the DOM (Document Object Model) data structure, which is difficult to handle, while a JSON document can be easily de-serialized into a main-memory variable of any object-oriented programming language.

For all these reasons, today most portals adopt JSON to make their data collections accessible through APIs (Application Programming Interfaces), each one based on their own data structures; for example, Twitter freely provides tweets, that can be further analyzed (Cuzzocrea, et al., 2015). The consequence is that different source data sets, even related to the same application domain, may present a different structure, even though they describe the same information (e.g., books on sale). Thus, analysts must first collect the data sets coming from different sources and, then, face heterogeneous data structures during the analysis process.

The need for flexible querying has been widely studied both in relational databases (Bordogna \& Psaila, 2008), and in XML Retrieval (Marrara \& Pasi, 2016). The aim was to overcome the inadequacy of both SQL and XQuery caused by the classical Boolean conditions (also said crisp conditions) on which they are founded that neither allow for inexact matching between query and data, nor provide a ranking mechanism of the retrieved results with respect to their relevance to the informative needs of the users.

Currently, NoSQL Document Stores, able to natively store and query collections of JSON documents, have become very popular, in particular MongoDB ${ }^{1}$ and $A W S$ DocumentDB ${ }^{2}$. However, although these systems are schema free by definition, their query languages still lack suitable constructs for flexibly retrieving heterogeneous JSON documents with high-level and analyst-oriented instructions. The J-CO framework was designed to provide a unique framework to integrate and transform data sets described as collections of

\footnotetext{
${ }^{1}$ https://www.mongodb.com/

2 https://aws.amazon.com/documentdb/
} 
(possibly geo-tagged) JSON documents, stored within NoSQL databases. The idea is that a unique query language (named J-CO-QL), whose execution engine is independent of storing technology but can seamlessly connect to several storage systems, is the solution to overtake the isolation of different database systems. Second, it can overtake the limitation of the query languages supported by each single system. Furthermore, the language provides native support to process geo-tagging of JSON documents (Bordogna, et al., 2017) (Bordogna, et al., 2018) (Psaila \& Fosci, 2018).

However, J-CO-QL is still a crisp language, i.e., conditions are still based on classical Boolean logic. In this work, we try to define a first proposal to transform J-CO-QL into a flexible query language, aimed at exploiting the flexibility offered by Fuzzy Set Theory to exploit imprecise (linguistic) concepts. The reason why we propose to extend the J-CO-QL language is simple: its flexibility in dealing with heterogeneous and possibly geo-tagged JSON documents, joined with the flexibility provided by Fuzzy Set Theory, may provide, in perspective, a very flexible language for querying and transforming collections of JSON documents.

The main advantage of this solution lies in the simplicity with which the user can express selection conditions based on vague concepts; furthermore, JSON documents are implicitly ranked on the basis of their closeness to user's wishes. In this paper, we present a first step towards such an extension, as a preliminary validation of the approach. In Section 2, we discuss the related work and present some basic concepts concerned with Fuzzy Set Theory. In Section 3, we briefly introduce the fundamentals of the J-CO-QL language. In Section 4, a (basic) extension to J-CO-QL with Fuzzy Set Theory concepts is proposed. Finally, Section 5 draws the conclusions.

\section{RELATED WORK}

Most relational database management systems adopt the Boolean logic to formalize queries. This means that a query condition can be either satisfied or not satisfied. By using Boolean logic, it is not possible to have a flexible semantics of relational operations, to express preferences and to rank query results. In many real-world situations, queries are expressed by humans by means of imprecise words. User's intention is not merely to find the tuples that satisfy a given query; in contrast, the user could wish to estimate how much each tuple satisfies the conditions in the query (its satisfaction degree), for possibly ranking tuples.

There are several approaches to represent imprecise and vague concepts in Information Retrieval (IR). A first approach defines similarity or proximity relations between pairs of imprecise and vague items. In the vector space model, for instance, documents and queries are represented as points in a space of terms and the distances between the points representing the query and the documents are used to quantify their similarity (Salton, 1994). Another category of approaches adopts the notion of Fuzzy Set. Fuzzy Set Theory is an extension to classical set theory (Zadeh, 1975). The notion of fuzzy set has been used to represent vague concepts expressed in a flexible query for specifying soft selection conditions (Rijsbergen, 1979). The objective here is to quantify the closeness of the information carried by the proposition with the considered reality. Possibility Theory (Fuhr, 1989; Zadeh, 1965) together with the concept of a linguistic variable defined within fuzzy set theory (Zadeh, 1975), provides a complete formal framework to manage imprecise, vague and uncertain information (Buell, 1985).

There are two alternative ways to model the retrieval activity. (i) One possibility is to model the query evaluation mechanism as an uncertain decision process. The concept of relevance is defined as a binary (crisp) condition, since the query evaluation mechanism computes relevance probability of a document $d$ with respect to a query $q$. Such an approach, which does model the uncertainty of the retrieval process, has been introduced and developed by using probabilistic IR models (Dubois \& Prade, 1988; Herrera \& Herrera-Viedma, 1997; Waller \& Kraft, 1979). (ii) Another way is to interpret the query as the specification of soft constraints that the representation of a document can satisfy to a certain degree, and to consider term relevance as a gradual (vague) concept. This is the approach adopted in fuzzy IR models (Bordogna \& Pasi, 1995). In this latter case, the decision process performed by the query evaluation mechanism computes the degree with which the query is satisfied by the representation of each document. A very good survey regarding the adoption of Fuzzy Sets in IR can be found in (Kraft, et al., 2015).

A well defined context, in which dealing with uncertainty and vagueness is quite common, is $X M L$ Retrieval. When defining query languages for XML documents, the problem of querying data collections without a well-defined structure, or with a heterogeneous structure, was soon evident. 
To tackle this problem, Fuzzy Set Theory was a fairly immediate choice. In (Damiani \& Tanca, 2000) the authors presented an approach in which XML documents are modeled as labeled graphs; their structure is selectively extended by computing fuzzy estimates of how much the information provided by XML tags is important. The results of such an extension are fuzzy labeled graphs. Query results are sub-graphs of these fuzzy labeled graphs, presented as a ranked list according to their matching degree with respect to the query.

In a different perspective, the approaches presented in (Campi, et al., 2009; Marrara \& Pasi, 2016) propose flexible XML selection languages, which allow for formulating flexible constraints on both structure and content of XML documents. In this way, users can formulate queries that are able to retrieve XML documents that provide information close to users' information needs.

According to many research papers (Kacprzyk \& Zadrożny, 1995; Medina, et al., 1994) there are two main ways to apply Fuzzy Set Theory in the context of database management systems. The first way consists in developing a fuzzy database model to manage imprecise data (Bosc \& Prade, 1997).

The second way develops a fuzzy querying interface on top of a conventional relational database (Bosc \& Prade, 1997); the advantage of this approach is evident in the SoftSQL proposal (Bordogna \& Psaila, 2009). Other extensions of SQL have been proposed: FSQL (Galindo, 2006) and SQLf (Bosc \& Pivert, 1995).

\subsection{A Brief Introduction on Fuzzy Sets}

Fuzzy Set Theory was introduced by Zadeh (Zadeh, 1975), and soon applied to different areas, such as artificial intelligence, natural language processing, decision making, expert systems, neural networks, control theory, and so on. In this section, we briefly review the main concepts, useful to understand the approach proposed in this paper.

From now on, we denote by $X$ a non-empty universe, either finite or infinite.

Definition 1: A fuzzy set (or type-1 fuzzy set) $A \subseteq X$ is a mapping $A: X \rightarrow[0,1]$. The value $A(x)$ is referred to as the membership degree of the element $x$ to the fuzzy set $A$.

Therefore, a fuzzy set $A$ in $X$ is characterized by a membership function $A(x)$ which associates each element $x \in X$ with a real number in the interval $[0,1]$; in other words, given $x$, the value $A(x)$ represents the degree of membership of $x$ to $A$.

A fuzzy set is empty if and only if its membership function is identically zero on $X$. Two fuzzy sets $A$ and $B$ are equal, written as $\mathrm{A}=\mathrm{B}$, if and only if $A(x)=B(x)$ for all $x \in X$.

The complement of a fuzzy set $A$ is denoted as $A^{\prime}$ and is defined by $A^{\prime}(x)=1-A(x)$.

As in the case of ordinary sets, the notion of containment plays a central role in the ease of fuzzy sets.

Definition 2: $A$ is contained in $B$ (or, equivalently, $A$ is a subset of $B$, or $A$ is smaller than or equal to $B$ ) if and only if $A(x) \leq B(x)$, for each $x \in X$.

Definition 3: The union (resp. intersection) of two fuzzy sets $A$ and $B$ with respective membership functions $A(x)$ and $B(x)$ is a fuzzy set $C$, written as $C=A \cup B$ (resp. $C=A \cap B$ ), whose membership function is related to those of $A$ and $B$ by $C(x)=\operatorname{Max}(A(x), B(x))($ resp. $C(x)=\operatorname{Min}(A(x), B(x)))$, for all $x \in X$.

The intersection is used to express the logical AND operator, the union formalizes the logical OR operator while the complement set represents the logical NOT operator.

\section{THE J-CO-QL LANGUAGE}

The J-CO-QL language is a rich language to query and transform collections of complex, possibly geo-tagged, JSON documents,. Here, we briefly introduce some basic constructs by means of an example. For a complete introduction to the language, the reader can refer to (Bordogna, et al., 2017) (Bordogna, et al., 2018) (Psaila \& Fosci, 2018). 
Suppose we have a collection named Hotels, stored within a database named MyDB. The documents in this collection describe hotels located worldwide. A sample document is reported in Listing 1.

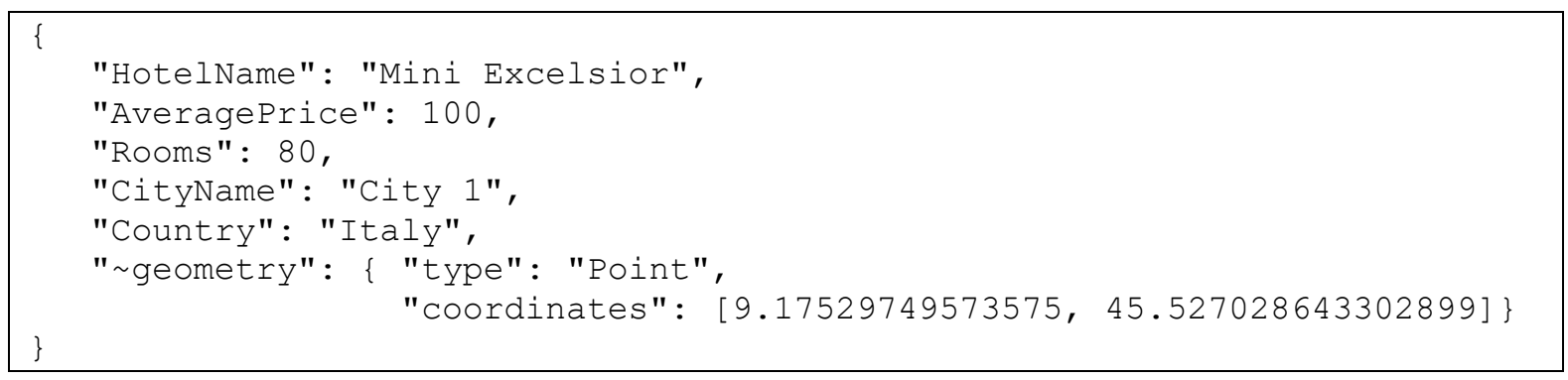

Listing 1. sample document in collection Hotels

Notice the presence of the geometry field: it is a standard JSON field describing the geometry (e.g., points, polylines or regions) of the document, by relying on the GeometryDefinition defined in the GeoJSON format ${ }^{3}$. However, it assumes a specific role in our language: if present, it is automatically processed by the spatial operations provided by J-CO-QL. In this paper, we do not focus on spatial operations; we will consider them in our further extensions of the language. Note that field names beginning with $\sim$ are fully compliant JSON field names; thus, any JSON document store is able to store them.

The following query generates a new collection, named ItalianHotels. Let us discuss it.

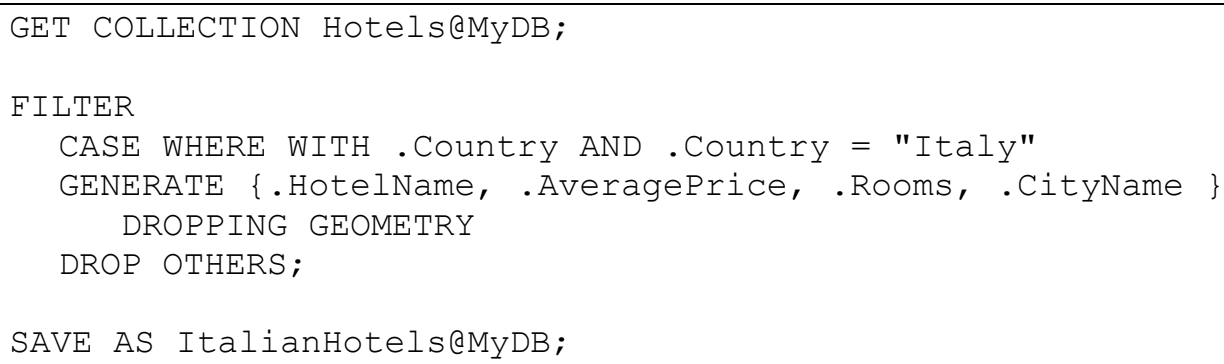

Listing 2. J-CO-QL query that creates a collection of documents describing Italian Hotels

The query reported in Listing 2 contains three instructions, performing either simple tasks or complex tasks. The underlying semantics is the following: each instruction starts from an Execution State $s_{i}$, and produces a new execution state $s_{(i+1)}$. Each execution state contains several information, including also the Temporary Collection, i.e., a collection of JSON documents that constitutes the input of the instruction. Thus, each instruction may use this collection and may generate a new temporary collection.

We can now describe the query in Listing2.

- The GET COLLECTION instruction retrieves the collection named Hotels from the database named MyDB. This collection becomes the temporary collection of the process.

- The FILTER instruction selects and transforms the documents in the temporary collection, generating a new temporary collection. In this case, since the temporary collection contains documents describing hotels, the CASE WHERE clause selects documents having the Country field (by means of the WITH predicate) whose value is "Italy".

The selected documents are restructured by the GENERATE action, in order to slightly simplify them. Specifically, we keep all fields except Country; furthermore, the option DROPPING GEOMETRY says that we do not want the geometry field in the output documents.

The DROP OTHERS option, at the end of the instruction, means that documents not selected by the CASE WHERE clause must be discarded from the new temporary collection produced by the instruction. Listing 3 shows a sample document appearing in the new temporary collection.

\footnotetext{
${ }^{3}$ https://tools.ietf.org/html/rfc7946
} 
- Finally, the temporary collection produced by the FILTER instruction (that contains only Italian hotels) is saved into the MyDB database with name ItalianHotels.

Notice the dot notation . Country to refer to fields: this is motivated by the fact that documents can contain nested documents and fields; the dot notation allows for referring to nested fields, for example .A.B (the initial dots means that the A field is root in the root level).

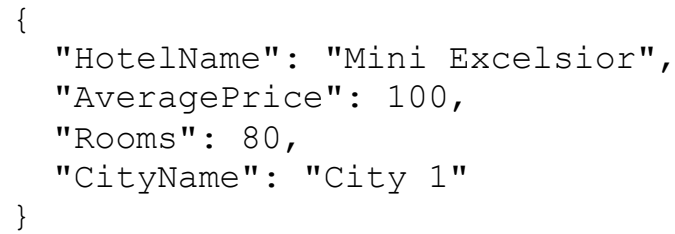

Listing 3. sample document in obtained after the JOIN instruction

\section{EXTENDING THE J-CO-QL LANGUAGE WITH FUZZY SETS}

We can now address the main goal of this paper, i.e., to propose an extension of J-CO-QL to support fuzzy sets. First of all, we have to extend the data model.

\subsection{Data Model}

J-CO-QL was designed to work on JSON documents. However, in order to manage geometries of geo-referenced documents, we had to introduce a special field, named geometry, which is implicitly considered by the instructions, but it is still a JSON-compliant field and can be accessed by expressions and conditions, irrespective of its particular role. Hereafter, we adopt a similar approach to represent fuzzy sets which documents belong to.

A JSON document $d$ can belong to one or more fuzzy sets $A_{l}, A_{2}, \ldots, A_{n}$. For each fuzzy set $\mathrm{A}_{i}, d$ belongs to $A_{i}$ with a certain membership degree $A_{i}(d) \in[0,1]$. To represent the multiple membership degrees of $d$, a special root-level field named fuzzysets is introduced: it is a nested document, containing only numerical fields; each field denotes the membership degree of the JSON document to the fuzzy set named as the field.

As an example, consider the following JSON document.

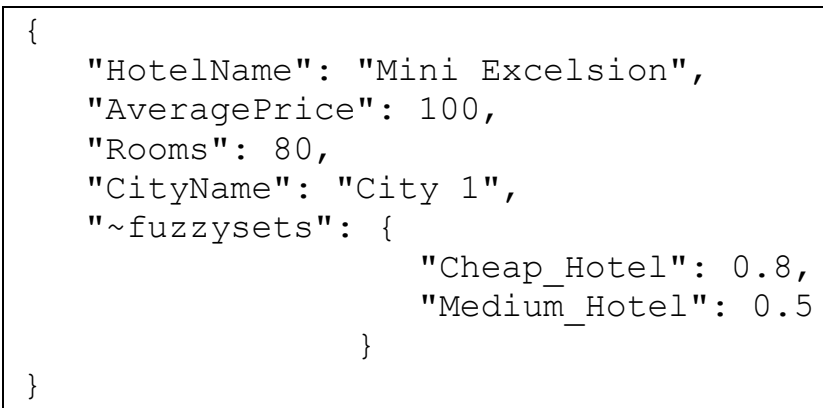

The document describes a hotel that belongs to two fuzzy sets named Cheap Hotel and Medium_Hotel. The membership degree is 0.8 for the Cheap_Hotel fuzzy set (for example we could say that with an average price less than 80 Euros the hotel would be considered as fully cheap, while with an average price of 100 Euros the hotel is considered partially cheap). The membership degree is 0.5 for the Medium_Hotel fuzzy set, meaning that the hotel is partially "medium", but not completely (for example, we could say that a hotel is fully medium with at least 40 rooms and at most 75 rooms).

Notice that the document does not say how the membership degrees of fuzzy sets were evaluated, but only that it belongs to some fuzzy sets with some membership degrees. 
The advantage of this solution is that the document is still a standard JSON document: it can be stored into database collections and retrieved from them.

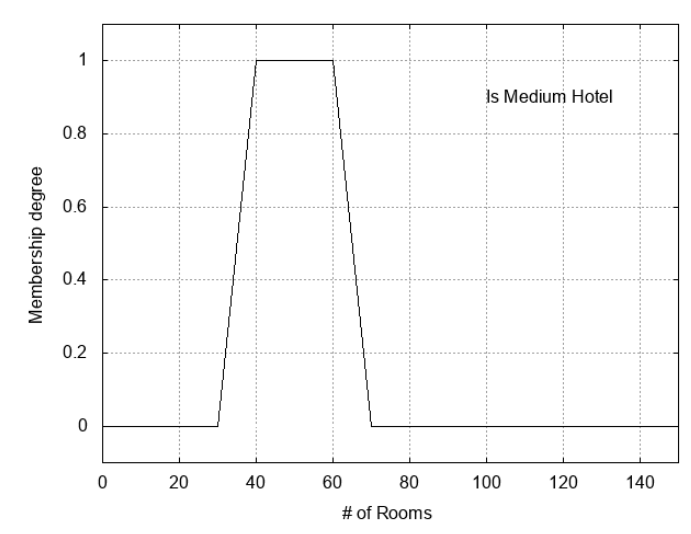

(a)

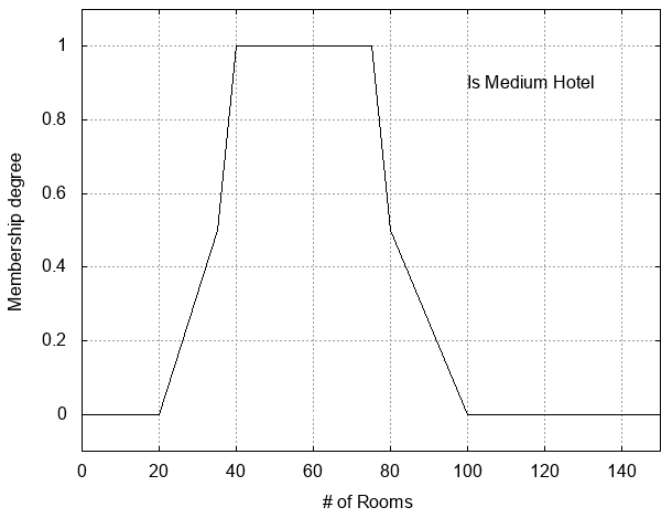

(b)

Figure 1. a) classical trapezoidal membership function and b) enhanced membership function as supported by J-CO-QL

\subsection{Creating Fuzzy Operators}

In order to evaluate if a document belongs to a fuzzy set, it is necessary to define some operators able to compute the membership degree of a document with respect to a fuzzy set.

A classical solution, proposed in previous literature on relational database extended with fuzzy sets, is to define a trapezoidal function in the codomain $[0,1]$ (see Figure 1.a, for an example). Here, we propose an extended interpretation of this approach, where we allow for the definition of an arbitrarily long polyline with codomain $[0,1]$, as reported in Figure $1 . b$, in order to model more complex real contexts.

The J-CO-QL language offers a new specific construct to create fuzzy operators.

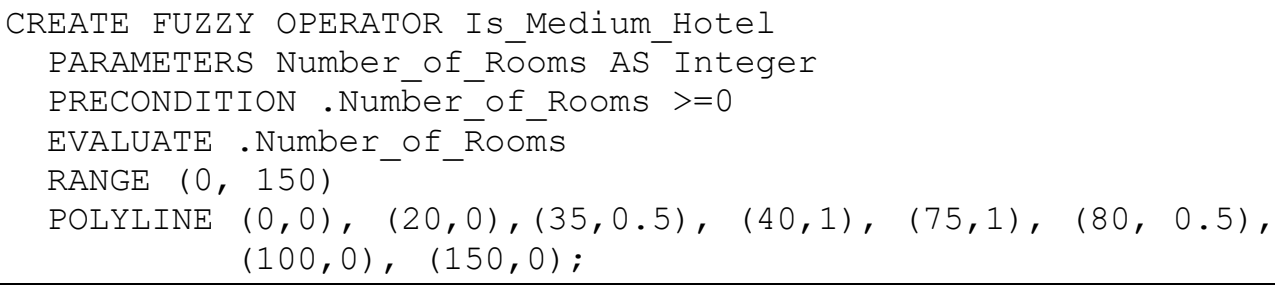

The construct defines the new fuzzy operator Is_Medium_Hotel. In the following, we explain the definition of the fuzzy operator.

First of all, the PARAMETERS clause specifies the formal parameters received by the operator. Specifically, the Is_Medium_Hotel operator receives one single parameter, named Number_of Rooms, definined as an integer value. When the operator is invoked, all parameters are assembled into one JSON document, having the formal parameters as fields and the actual parameters as values. In our case, this JSON document will have only the Number_of_Rooms field.

In order to obtain the membership degree, we have to evaluate an expression based on these parameters. The EVALUATE clause specifies this expression. In our case, the value of the Number_of Rooms parameter is kept untouched. Notice that we still use the dot notation. Number_of Rooms to refer to the parameters (as in the other instructions of J-CO-QL): in fact, the expression actually works on the JSON document obtained by assembling formal and actual parameters. 
However, not all values of the input parameters are valid: the PRECONDITION clause specifies a condition to be verified before performing the evaluation. If the condition is false, no membership value is generated and the operator generates an evaluation error.

The RANGE clause specifies the range within which the result of the EVALUATE expression is mapped to the corresponding membership degree, by means of the polyline function defined in the subsequent POLYLINE clause. In this case, we consider a range between 0 and 150 rooms.

Finally, the POLYLINE clause defines the polyline used as membership function. It is defined as a sequence of pairs $(x, y)$, where $x$ is a coordinate in the range specified by the RANGE clause, while $y$ is a value specified in the range $[0,1]$. The first pair has the value of the $x$ coordinate that coincides with the left bound of the range, while the last pair contains a value for the $x$ coordinates that is equal to the right bound of the range. This way, it is possible to define the membership function as a polyline, where the $y$ coordinates denotes the final membership value.

For example, the membership function for the Is Medium Hotel operator is depicted in Figure 1.b: notice the points of the polyline that correspond to the sequence of pairs. If we suppose that the actual value of the Number_of_Rooms parameter is 50, the corresponding y coordinate is 1 (the membership degree), i.e., the hotel fully belongs to the set of medium hotels; if the value for the Number of Rooms parameter is 10 , the corresponding membership degree is 0 , meaning that the hotel does not belong at all to the set of medium hotels. And so on.

We assume that in case of values of the expression specified in the EVALUATE clause that are lower than the left bound (greater than the right bound, resp.), the $y$ value of the left bound (right bound, resp.) is the membership value. For example, if the Number_of_Rooms parameter is 200, the membership value is 0 .

\subsection{Extending the FILTER Operator}

Consider the ItalianHotel collection generated by the query reported in Listing 2 (a sample document in this collection is reported in Listing 3). We want to evaluate the degree with which hotels belong to the fuzzy set named Medium_Hotel. The following J-CO-QL query does this work.

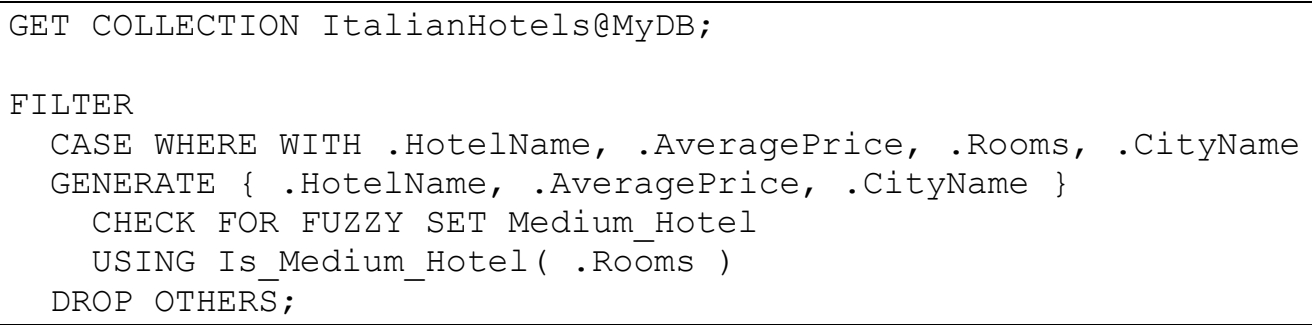

Listing 5. J-CO-QL query to create the Medi um_Hotel fuzzy set

The above query contains two instructions. First of all, the GET COLLECTION instruction retrieves the ItalianHotels collection from the MyDB database.

Then, the FILTER instruction transforms the documents in the temporary collection. If a document satisfies the WHERE condition in the CASE clause, it is processed and will appear in the output temporary collection, otherwise it is discarded (as specified by the DROP OTHERS option).

The WHERE condition is satisfied if the document contains the fields named HotelName, AveragePrice, Rooms and CityName. If so, it is restructured by the GENERATE action, by keeping only fields HotelName, AveragePrice and CityName.

At this point, the novel CHECK FOR FUZZY SET action adds the new fuzzy set to the output document. The fuzzy set is named Medium_Hotel; its membership degree is evaluated by the fuzzy logic condition reported in the USING sub-clause. The fuzzy logic condition can contain fuzzy operators, fuzzy set names and classical crisp comparison predicates (in this case, the membership degree 1 corresponds to true, while the membership degree 0 corresponds to false), connected by the fuzzyfied AND, OR and NOT operators. 
In the instruction, the fuzzy condition specified in the USING sub-clause simply exploits the Is_Medium_Hotel operator previously defined, by using the Rooms field of the current document as actual value of the Number of Rooms formal parameter. Since the documents in the source temporary collection do not belong to any fuzzy set, the fuzzysets field is created; within it, a unique field named Medium_Hotel is present, whose value is the degree with which the document belongs to the fuzzy set (the membership degree is obtained by evaluating the fuzzy operator Is_Medium_Hotel). Listing 6 reports the document obtained by transforming the document depicted in Listing 3.

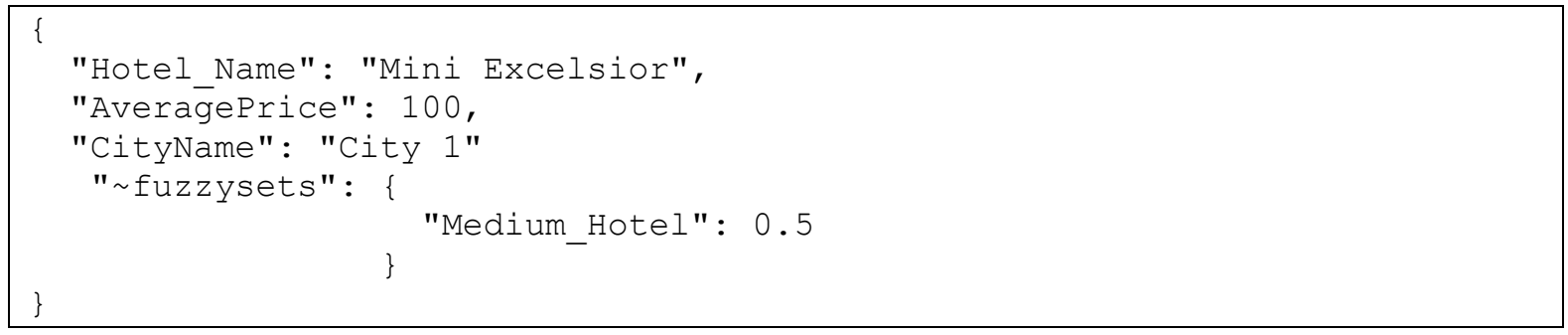

Listing 6. Sample document belonging to one fuzzy set

To appreciate the usefulness of the new constructs, consider Listing 7 extending the querying Listing 5.

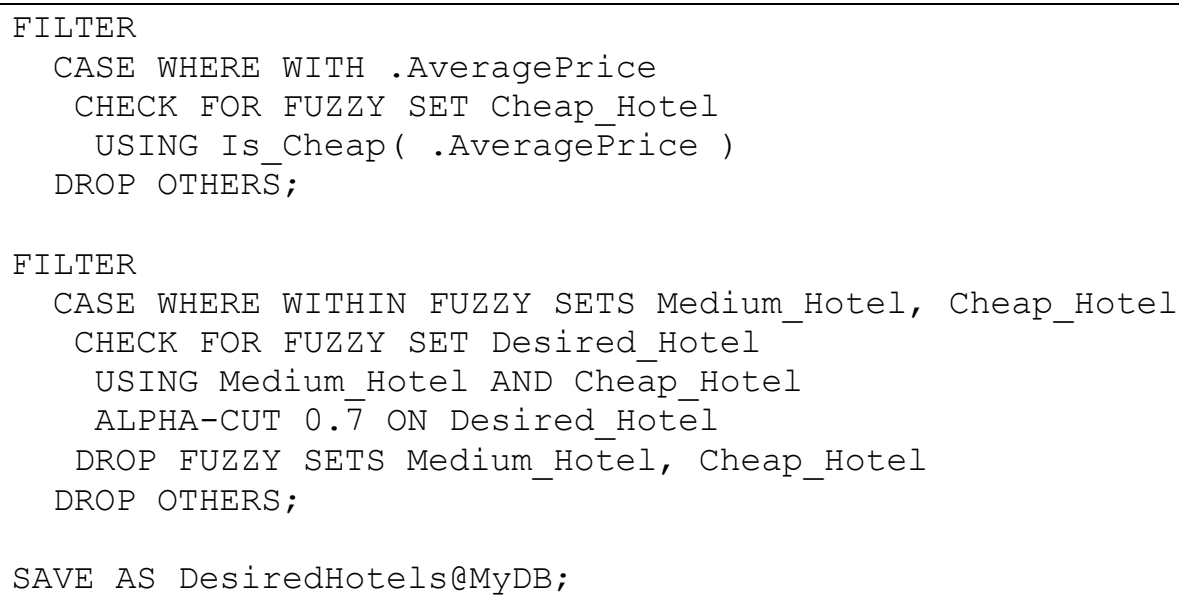

Listing 7. Second part of the J-CO-QL query based on fuzzy sets

Suppose we have defined another fuzzy operator named Is_Cheap_Hotel, whose goal is to evaluate if a hotel is cheap on the basis of its average price per night. The first FILTER instruction reported in Listing 7 further extends documents in the temporary collection by adding a new fuzzy set, named Cheap_Hotel. The resulting documents now contain two fuzzy sets, i.e., Medium_Hotel and Cheap_Hotel. Notice that the GENERATE action is missing: this means that the structure of documents into the output temporary collection does not change.

At this point, we want to focus only on hotels of interests. For example, we would like to find out hotels that are both medium hotels and cheap.

The second FILTER instruction in Listing 7 evaluates a third fuzzy set, named Desired_Hotel: as specified in the USING fuzzy condition, a hotel must be both in the Medium Hotel fuzzy set and in the Cheap_Hotel fuzzy set. This is expressed by the fuzzy AND condition (remember that the resulting membership degree is the minimum one). However, we are not interested in all hotels, but only in those whose membership degree to the Desired_Hotel fuzzy set is at least 0.7: the ALPHA-CUT specification discards documents with lower membership degree.

Furthermore, the DROP FUZZY SETS sub-clause specifies the fuzzy sets that are removed from the output documents (thus, only the Desired Hotel fuzzy set appears in the output documents). 
Notice the presence of the WITHIN FUZZY SETS predicate in the WHERE condition: this is a novel crisp predicate that is true if a document has a membership degree greater than 0 for the specified fuzzy sets.

The query terminates by saving the temporary collection into the MyDB database with name DesiredHotels.

The reader can see that by evaluating the belonging of documents to various fuzzy sets, it is possible to filter documents by expressing soft conditions, based on linguistic concepts (medium and cheap). Furthermore, the final membership degree could be also used to sort the results in reverse order of importance. This shows that the proposal, although it is the early stage of the extension of J-CO_QL to support fuzzy concepts, seems to be on the right way.

\section{CONCLUSIONS}

In this work, we propose a first solution to the problem of how to query heterogeneous collections of JSON documents in a flexible way, by means of vague concepts. The aim of the paper is to propose a first extension of the J-CO-QL language: we try to define a flexible query language, aimed at exploiting the flexibility offered by Fuzzy Set Theory to exploit imprecise (linguistic) concepts or to deal with uncertainty. The J-CO-QL language, due to its flexibility in dealing with heterogeneous and possibly geo-tagged JSON documents, joined with the flexibility provided by Fuzzy Set Theory, may become, in perspective, a very flexible language for querying and transforming collections of JSON documents.

In this paper, we presented a first step towards such an extension, as a preliminary validation of the approach. Future work will further extend this preliminary proposal into a powerful and complete fuzzy query language, also providing a complete formal definition. We will also test efficiency, in order to evaluate the overhead caused by the introduction of fuzzy constructs.

\section{REFERENCES}

Bordogna, G., Capelli, S., Ciriello, D. E. \& Psaila, G., 2018. A cross-analysis framework for multi-source volunteered, crowdsourced, and authoritative geographic information: the case study of volunteered personal traces analysis against transport network data. Geo-spatial Information Science, 21(3), pp. 257-271.

Bordogna, G., Capelli, S. \& Psaila, G., 2017. A Big Geo Data Query Framework to Correlate Open Data with Social Network Geotagged Posts. The Annual International Conference on Geographic Information Science (AGILE 2017), Wageningen, The Netherlands. Springer, Cham, pp. 185-203.

Bordogna, G. \& Pasi, G., 1995. Linguistic aggregation operators of selection criteria in fuzzy information retrieval. Int. Journal of Intelligent Systems, 10(2), pp. 233-248.

Bordogna, G. \& Psaila, G., 2009. Soft Aggregation in Flexible Databases Querying based on the Vector p-Norm. International Journal of Uncertainty, Fuzziness and Knowledge-Based Systems, Volume 17, pp. 25-40.

Bosc, P. \& Pivert, O., 1995. SQLf: a relational database language for fuzzy querying. IEEE Transactions on Fuzzy Systems, 3(1), pp. 1-17.

Bosc, P. \& Prade, H., 1997. An Introduction to the Fuzzy Set and Possibility Theory-Based Treatment of Flexible Queries and Uncertain or Imprecise Databases. Uncertainty Management in Information Systems, pp. 285-324.

Buell, D. A., 1985. A problem in information retrieval with fuzzy sets. Journal of the Association for Information Science and Technology, 36(6), pp. 398-401.

Campi, A. Daminai, E., Guinea, S., Marrara, S., 2009. A fuzzy extension of the XPath query language. intelligent information systems, 33(3), pp. 285-305.

Cuzzocrea, A., Psaila, G. \& Toccu, M., 2015. Knowledge Discovery from Geo-Located Tweets for Supporting Advanced Big Data Analytics: A Real-Life Experience. model and data engineering, Volume 9344, pp. 285-294.

Damiani, E. \& Tanca, L., 2000. Blind queries to XML data. Lecture Notes in Computer Science, pp. 345-356.

Dubois, D. \& Prade, H. M., 1988. Possibility Theory: An Approach to Computerized Processing of Uncertainty. s.l.:s.n.

Fuhr, N., 1989. Models for retrieval with probabilistic indexing. Information Processing and Management, 25(1), pp. 55-72.

Galindo, J., 2006. Fuzzy Databases: Modeling, Design, and Implementation. s.l.:s.n. 
Herrera, F. \& Herrera-Viedma, E., 1997. Aggregation operators for linguistic weighted information. systems man and cybernetics, 27(5), pp. 646-656.

Kacprzyk, J. \& Zadrożny, S., 1995. Fquery for Access: Fuzzy Querying for a Windows-Based DBMS. , pp. 415-433.

Kraft, D. H., Colvin, E., Bordogna, G. \& Pasi, G., 2015. Fuzzy Information Retrieval Systems: A Historical Perspective. Fifty Years of Fuzzy Logic and its Applications, pp. 267-296.

Marrara, S. \& Pasi, G., 2016. Fuzzy Approaches to Flexible Querying in XML Retrieval. International Journal of Computational Intelligence Systems, 9(100), pp. 95-103.

Medina, J. M., Pons, O. \& Vila, M. A., 1994. GEFRED: a generalized model of fuzzy relational databases. Information Sciences, 76(1), pp. 87-109.

Psaila, G. \& Fosci, P., 2018. Toward an Anayist-Oriented Polystore Framework for Processing JSON Geo-Data. International Conferences on WWW/Internet, ICWI 2018 and Applied Computing 2018, Budapest; Hungary, 21-23 October 2018, pp. 213-222.

Rijsbergen, C. J. V., 1979. Information Retrieval (2nd ed.). Newton, MA, USA: Butterworth-Heinemann .

Salton, G. e. a., 1994. Automatic analysis, theme generation, and summarization of machine-readable texts.. Science, 264(5164), pp. 1421-1426..

Waller, W. \& Kraft, D. H., 1979. A mathematical model of a weighted boolean retrieval system. Information Processing and Management, 15(5), pp. 235-245.

Zadeh, L., 1975. The concept of a linguistic variable and its application to approximate reasoning-I. Information Sciences, 8(3), pp. 199-249.

Zadeh, L. A., 1965. Fuzzy Sets. Information and Control, Volume 8, pp. 338 - 353. 